\title{
JARDINES TERAPÉUTICOS
}

\author{
TERAPEUTIC GARDENS
}

Cinzia Mulé

\section{RESUMEN}

Siempre se piensa en el jardín como un lugar puramente lúdico, como puede ser un parque o el simple jardín de una habitación. Muy pocas veces se piensa en el poder de sanación que pueden tener las plantas ornamentales, que efecto tienen dentro de una estructura sanitaria? Desde los años 80 se vienen estudiando los efectos que producen en los enfermos y se ha demostrado científicamente los resultados positivos que se obtienen. Su uso se hace cada día más indispensable para bajar los costes y días de permanencia de los pacientes en los hospitales, y mejorar la calidad de vida de los enfermos en estructuras donde necesitan de cuidados a largo plazo.

Los elementos fundamentales con los que un paisajista puede contar y debería tener en consideración para realizar un buen proyecto son el tema de este artículo.

\section{Palabras clave}

Jardines terapéuticos, horticultura, jardines restaurativos, jardines de rehabilitación, jardines rituales, espacios contemplativos, reducción del estrés.

\section{ABSTRACT}

Gardens have always been considered only as purely recreational places, like parks or simple garden rooms. The healing power that ornamental plants may have is rarely considered. What effects do they have when located in a medical facility? Those effects have been studied since the $80 \mathrm{~s}$ and the positive results obtained are scientifically proven. Their use is becoming more indispensable each day in order to lower costs and the hospitalization time of patients, and also to improve their life quality when long-term care is required.

The fundamental elements that a landscape designer must know and should take into account to make a good project are the subject matter of this paper.

\section{Keywords}

Therapeutic garden, horticulture, restorative garden, rehabilitation garden, ritual garden, contemplative spaces, stress reduction

1 Se licenció en Arquitectura en la Universidad Particular Ricardo Palma en 1980, en la Universidad de Génova en el 1994. Máster en Paisajismo en Génova en 1984. Beca del Ministero degli Affari Esteri italiano desde el 81-84. Inscrita al Colegio de Arquitectos de Lima desde 1981. Inscrita en el AIAPP (Associazione Italiana di Architettura del Paesaggio) desde el 1986. Inscrita al Ordine di Architetti di Roma desde 1995. 


\section{INTRODUCCIÓN}

De qué manera se puede definir un 'Jardín Terapéutico' es lo que trataré de explicar en este breve artículo, desde su definición, una breve visión a través de la historia, los beneficios que genera y sobre todo cuales son los elementos fundamentales con los que un paisajista puede contar y debe tener en consideración para la realización de su diseño.

La más simple definición es la de un espacio exterior diseñado para que las necesidades físicas, psicológicas, sociales y espirituales de las personas internadas en una estructura sanitaria, las ayude a mantener contacto con la realidad y proporcione bienestar psicofísico a los usuarios, por lo tanto, pacientes, visitantes y personal sanitario.

Una definición un poco más compleja es la que nos da Clare Cooper Marcus, en la que presenta al jardín terapéutico 'no como un lugar sino como un concepto', es decir el resultado de una comunión entre el paisajista y el médico.

\section{ENFOQUE HISTÓRICO}

Entre los años 1950 a 1990 se pierde completamente el concepto del valor terapéutico de la naturaleza en la mayor parte de los hospitales occidentales. Predomina el 'internacional style', con la construcción de edificios hospitales semejantes a torres de oficinas, con ventilación e iluminación artificial en los dormitorios, donde no existe el contacto con el exterior, incluso en las salas de espera. Donde los espacios exteriores son de uso exclusivo para las maquinarias o dedicados a estacionamientos. La 'productividad' de la institución está basada en la ganancia y eficiencia en muchos casos, perdiendo la empatía con el paciente. Ya no se trata de dar un servicio a la comunidad, son y funcionan como empresas en donde la ganancia tiene prioridad.

Sin embargo, en los siglos pasados y hasta antes de 1950, los jardines en los hospitales fueron considerados fundamentales en la recuperación del enfermo. Que pudiese disfrutar del buen aire, del sol y de las propiedades de sanación de algunas plantas siempre fueron elementos importantes.

En la Edad Media los claustros de los monasterios, que en muchos casos eran hospitales, brindaban un lugar seguro, a escala humana. El paciente podía descansar en el pórtico al cubierto, en el patio-jardín donde muchas veces había árboles y ampararse bajo su sombra si fuese necesario, caminar por los senderos, sentarse en el césped y bancas.

Lo describe San Bernardo (1090-1153), haciendo referencia a sus pacientes en el Hospice de Clairvaux en Francia, relatando los beneficios que gozan al permanecer al aire libre, estimulando los cinco sentidos y reconfortando a las personas enfermas o inválidas.

El concepto de estos jardines meditativos y regeneradores se pierde con la decadencia de los hospitales-monasterios en los siglos XIV y XV. La sanidad queda básicamente en manos de la autoridad eclesiástica y la mayor preocupación era la de construir grandes salas desde donde todos los pacientes pudiesen seguir la misa. Como, por ejemplo, el Ospedale Maggiore di Milano que tenía una planta a cruz como una iglesia y en los dormitorios las ventanas eran altas y los enfermos no podían ver el jardín.

Sin embargo, en muchos hospitales la tradición del patio no se perdió. Como por ejemplo, en Les Invalides de Paris, además, en muchos hospitales ingleses, en Marsella, Pisa, Constantinopolis, Trieste, Viena y Florencia.

Hay que llegar a los siglos XVII y XVIII cuando se vuelve a tener en cuenta la importancia de la higiene, el aire fresco y la ventilación cruzada, para que se vuelva a considerar la importancia del espacio exterior como curativo.

Es Roger Ulrich, (1984) uno de los primeros estudiosos sobre la importancia de las áreas verdes en los sanatorios, que menciona en su obra a Christian Cay Lorenz Hirschfeld, 
teórico alemán experto horticultor del siglo XVIII, que describe los beneficios que gozan las personas internadas al entrar en contacto con la naturaleza:

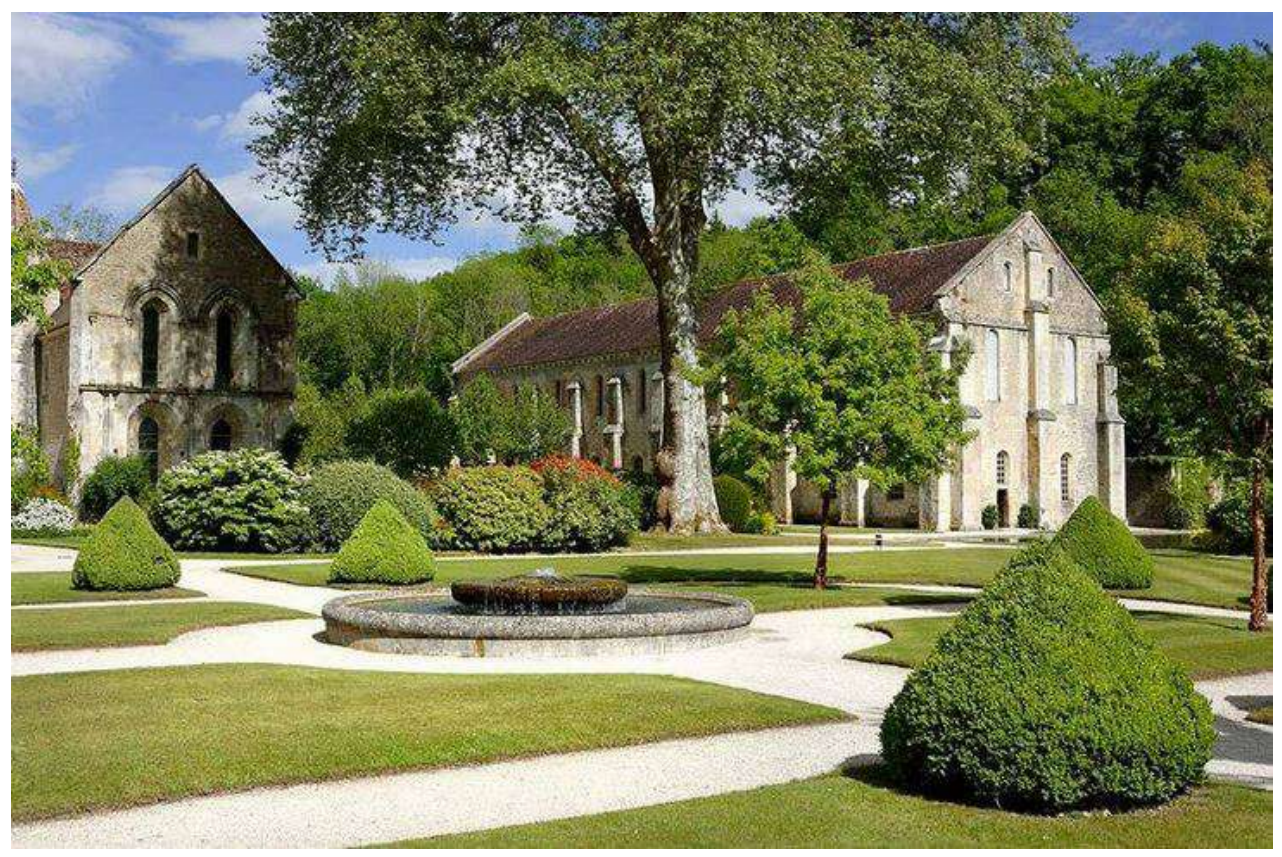

Hospice de Clairvaux

El hospital tiene que ser abierto, no encajado entre altas paredes. El jardín tiene que estar directamente conectado con el hospital o al límite muy cerca de él. Porque la vista de las flores $y$ los escenarios alegres vigorizan al paciente, la vista del jardín los alienta a querer caminar....además el césped debe tener senderos secos con sillas y bancas.....El jardín de un hospital debe tener todo lo necesario que incentive una vida saludable. Te debe ayudar a olvidar la debilidad y las preocupaciones y fomentar una actitud positiva....Los espacios tienen que tener césped y matas de flores.... Pequeños y ruidosos arroyos que corran entre matas de flores y las ruidosas cascadas entre arbustos llegar a nuestros oídos. Haciendo grupo con muchas plantas para fortalecer los aromas. El canto de los pájaros que encuentran reparo en los árboles alentarnos con su canto regocijando nuestro corazón. (Cooper, 1995, p. 8)

Todos conocen las importantes reformas que se realizaron en los hospitales ingleses en el siglo XIX gracias a Florence Nightingale (18201910), gran defensora de la higiene, la primera en usar un método científico y estadístico en la atención de los pacientes y en crear las bases para lo que sería la moderna enfermería. Para ella, eran fundamentales el aire libre, los jardines con flores, los dormitorios bien aireados e iluminados y las cabeceras de las camas cerca de las ventanas.

A fines del siglo XIX e inicios del XX, nacen los sanatorios para curar la tuberculosis y las camas con ruedas que permiten trasladar a los pacientes cerca de los jardines y pórticos, de manera que pudiesen tomar sol y aire puro.

Otro tipo de estructura sanitaria que desde el siglo XVIII y XIX hace uso del espacio exterior son los sanatorios de enfermos mentales en los cuales en muchos casos se comienza a usar la jardinería y agricultura dentro de sus regímenes de terapia..

Conelavanzardelamedicinayeldescubrimiento de los antibióticos, la importancia del contacto con la naturaleza comienza a perder su trascendencia. Se mantiene prevalentemente 
en estructuras que tratan a pacientes crónicos, o en aquellos que necesitan cuidados a largo plazo o en tratamiento psiquiátrico. La horticultura comienza a usarse como terapia para la recuperación de los veteranos de la Segunda Guerra Mundial.

Solo después de 1990, en Estados Unidos de América gracias al movimiento Patient Centered Care las administraciones se dan cuenta de lo negativo que son los ambientes institucionales. Se cambia de ruta y en un afán, diríamos competitivo, se comienza a pensar más en las necesidades de los pacientes, por lo cual se construyen edificios y entornos más acogedores y familiares.

Desde el año 2000, con la nueva reforma sanitaria en USA, se ha comenzado a medir el proceso de una administración a través del tiempo que el paciente permanece en el hospital (cuanto menor es el tiempo, es mejor) y la eficiencia del servicio. En consecuencia a partir de los estudios que comenzó Roger Ulrich en 1984, los realizados por Clare Cooper Marcus (1995) y Adrian Burton que publica los resultados de su investigación en "The Lancet Neurology"(2014), entre otros, se podría decir que son casi 30 años que se estudia e investiga sobre los efectos $y$ beneficios de los jardines terapéuticos construidos en estos años o simplemente los efectos que las áreas verdes ofrecen a los pacientes de los hospitales, hospicios, casas de cura o cualquier otra institución donde haya una patología física o psíquica que sanar.

\section{BENEFICIOS}

Como bien dice Clare Cooper Marcus, "un jardín no puede sanar una pierna rota o un cáncer" (Cooper, 2007, p. 4 ) o como dice Stephen Mitrione "un jardín terapéutico puede proporcionar alivio de la angustia psicológica causada por una enfermedad, pero no curar la misma" (Burton: 2014, p. 447-448)

Es decir está comprobado que puede:

reducir el estrés ayudando al cuerpo a encontrar su proprio equilibrio; reducir el dolor;

reducir la depresión, especialmente si incentiva el movimiento;

- mejorar la calidad de vida en los enfermos crónicos o terminales;

- ayudar al paciente a evocar sus propios recursos de sanación;

- ayudar al paciente a aceptar su condición de incurable;

- crear un ambiente adecuado donde el paciente pueda realizar terapias físicas, horticulturales, etc.;

- ser un espacio donde el personal pueda descansar en sus pausas laborales $y$ desestresarse, con un aumento en la producción y la calidad del servicio;

- disminuir el costo en algunas categorías de hospitalizados, reduciendo el tiempo de hospitalización y el uso de analgésico;

- ser un espacio donde se puedan reunir o encontrar los pacientes con sus familiares en un ambiente menos institucional que un hospital.

El principal cambio que denotan las personas después de haber transcurrido un tiempo en un jardín es el cambio de humor, relajamiento y sentimiento de serenidad. Ulrich fue el primero en realizar estudios relacionados a la visión de paisajes relajantes. Al principio consistieron simplemente en sustituir cuadros, paneles o fotos en las salas de espera, consultorios o dormitorios con imagines de paisajes naturales y relajantes, pasando con el poner plantas naturales en espacios comunes, en terrazas, en balcones, creando visuales panorámicas hacia el exterior hasta llegar a patios y jardines.

Ulrich en su primer estudio en 1984 trata sobre la relación del tiempo de hospitalización, el uso de analgésicos y la posibilidad de ver la naturaleza a través de una ventana del hospital en pacientes de un departamento de cirugía. Todas las investigaciones han sido documentadas durante los años 1981 hasta el 1992, seguidas por Hartig entre el 1991 al 1996 y en paralelo con las de Clare Marcus y Barnes en 1994. Estos últimos son los primeros en hacer un estudio sistemático en cuatro jardines de hospitales ubicados en la bahía de San Francisco. 
Los resultados de este último estudio son los siguientes:

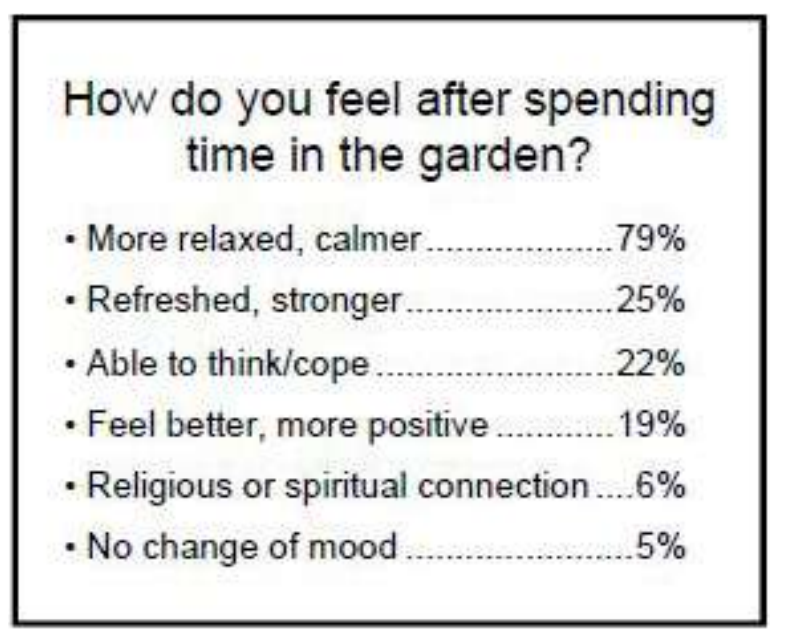

\section{What is it about the garden that helps you feel better?}

- Trees, plants, nature $59 \%$

- Smells, sounds, fresh air $58 \%$

- Place to be alone or with friend ...50\%

- Views, sub-areas, textures $26 \%$

- Practical features, benches, etc. . $17 \%$

- Don't know $.8 \%$

Responses from 143 garden users at four San Francisco Bay Area hospitals (Cooper Marcus, C. and M. Barnes, 1995).

En Italia a partir del 1998 hasta el 2015, se han realizado 24 'healing gardens'. Siete específicamente en hospicios que tratan el Alzheimer, cuatro en estructuras psiquiátricas, cinco en hospitales donde se trata específicamente el cáncer, uno para ciegos o discapacitados visuales, dos en estructuras neuro rehabilitativas, uno para pacientes terminales de HIV/AIDS y cuatro para cualquier tipo de patologías, pero principalmente cerca de unidades pediátricas. Las dimensiones y tipologías son diferentes, desde las terrazas de simplemente $20 \mathrm{~m}^{2}$ hasta parques de $7000 \mathrm{~m}^{2}$. En muchos casos, la necesidad de realizar estos jardines nace de la sensibilidad de los mismos médicos responsables.

Este es el caso de las terrazas terapéuticas realizadas en el 'Dipartimento Oncolngico dell 'Ospedale di Carrara', donde gracias a los doctores Maurizio Cantore y Andrea Mambrini se crea un grupo de trabajo que en el 2007 realiza dos pequeñas terrazas, cada una de $20 \mathrm{~m}^{2}$. Espacios que con la participación de los pacientes, doctores, técnicos y arquitectos paisajistas se ha tratado no solo de satisfacer las exigencias de los usuarios, sino que ha sido estudiado y los resultados presentados en el 2011.
El objetivo de este estudio era el de verificar los efectos que producían los 'jardines terapeúticos' en los pacientes hospitalizados del departamento de oncología, evaluando los parámetros psicológicos, vitales y la cantidad de medicinas relacionados con el estrés. Además se incluye una evaluación económica sobre el gasto farmacéutico debido al uso de ansiolíticos, analgésicos y antieméticos. Como se podrá ver en el siguiente cuadro que presento, se tuvieron grandes ventajas en lo que se refiere el ahorro de medicamentos, bienestar no solo en los pacientes sino una mayor productividad del personal $y$, en consecuencia, mayor calidad en el servicio.

Aunque todas estas ventajas han ayudado a que el sistema sanitario nacional (en Italia) considerara $y$ viese como positivo la introducción, donde fuese posible, de proyectos de esta naturaleza. Sin embargo, después de un terremoto en la zona de Carrara, por un probable riesgo estructural en las terrazas, parte del proyecto fue reconsiderado y desmantelado. Pero se podría decir que en su totalidad fue un gran éxito, sea en la metodología participativa usada para la protestación, la realización y el uso, como por el método científico empleado para medir el objetivo que se había prefijado. 


\section{Therapeutical Terraces in Oncology at Carrara (IT)}

interdisciplinary team:

ASL 1 at Massa Carrara:

Maurizio Cantore, oncology, dir. Dep. Oncology

Massimo Orlandi, data manager, Oncology

Roberta Tartarini, blology, Oncology

Stefania Baldassarrt, fharmacist Pharmacy

University of Perugia, School of Agraria

Alvaro Standardi, Full Professor di arboricolture

Enrica Bizzarri, designer and hystoric of gardens,

instructor

ANVE (Associazione Nazionale Vivaisti Esportatori)

Maurizio Lapponi, nurseryman

Franco Pirone, landscape archictect

Elisabetta Margheriti nurseryman, journalist

Ambra Pedretti, UK UNESCO process phases:

interdisciplinary team set, identification of fields of interest

definition of modes and times

surveys; meetings with staff and inpatientsi

preparatory meetings and agreement signature

bechnical choices with office

choice of typology of plants

definition of metrics to compare

questionnaire for patients and relatives

separate design for the two terraces

general meeting to define project

book for patients' comments

identification of managoment encharged employees

\section{guidelines}

definition of the project and plant species

definition of the maintenance calendar

definition for metrics to highlight levels of approval

assessment of eventual differential therapeutical effects

chance to realize changes in progress

identifying and realizing of scientific assessment of therapeutic values
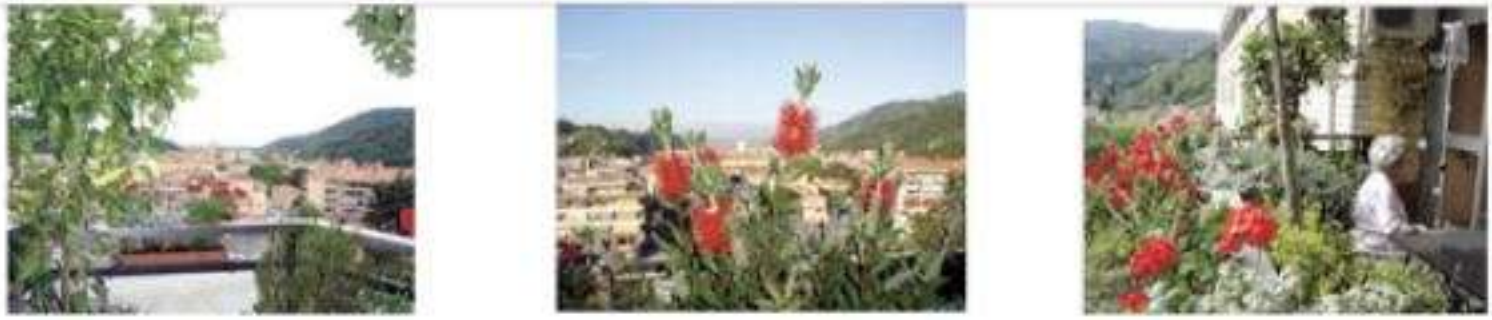

\section{Results on variation} in the consumption of drugs on 434 monitored patients

\begin{tabular}{|c|c|c|c|}
\hline Type of drings & $\begin{array}{l}\text { Consumption } \\
\text { during semester } \\
\text { before use of } \\
\text { terrace }\end{array}$ & $\begin{array}{l}\text { Consumpton } \\
\text { dreing semester } \\
\text { after vise of } \\
\text { terrace }\end{array}$ & $\begin{array}{l}\text { Percentage } \\
\text { of reduction }\end{array}$ \\
\hline antemescs (vals) & 10.8 & 9.08 & $-16 \%$ \\
\hline andinflammatones (vials) & 2.8 & 1,3 & $.53 \%$ \\
\hline anvolytics (vids) & 2,0 & 2,0 & \\
\hline anwolytics (tablest) & 5,3 & 0.2 & $.96 \%$ \\
\hline transdermal opiclds & 0,7 & 0.7 & \\
\hline orally opioids & 3.8 & 4,9 & $.15 \%$ \\
\hline
\end{tabular}

About one year after realization, the Company has eliminated part of the facility for measures against earthquake risk. The terraces are still in use: after the first ex-post evaluation, a new one is still ongoing. 


\section{TIPOLOGIA DE TERAPÉUTICOS \\ JARDINES}

Si bien no existe una clasificación sobre una tipología de Jardines Terapéuticos, por las experiencias realizadas en estos 30 años los podríamos clasificar de la siguiente manera:

1. Por el grado de actividad que se realiza en él.

2. Al servicio de una determinada patología médica.

3. Según el espacio a los que se tiene que adecuar.

4. Según el tipo de diseño en el que se inspira el paisajista.

\section{Por el grado de actividad que se realiza en el jardín terapéutico}

Esto nos hace pensar que en realidad un jardín puede inspirar una serie de acciones que van desde la más completa pasividad hasta una actividad enérgica. Por esto, las siguientes actividades las podemos englobar en dos grupos:

\subsection{Los de uso pasivo}

- El poder admirar un jardín desde una cama a través de la ventana

- Sentarse en una banca y admirar la naturaleza

- Poder meditar, rezar, hacer una siesta

- Leer, comer, conversar, escribir

En este grupo podríamos incluir los que se llaman Jardines restaurativos, de meditación o rituales. Son espacios meramente contemplativos, con la principal función de reducir el estrés y dar un soporte emocional aumentando la sensación de bienestar.

\section{- Jardines meditativos}

Generalmente es un espacio pequeño, muy tranquilo, contemplativo y específicamente dedicado a la meditación, pero como lo dice su mismo nombre no incluye otra actividad. Para evitar el efecto pecera (sentirse encerrado en un acuario) sería preferible que no estuviese rodeado por habitaciones.

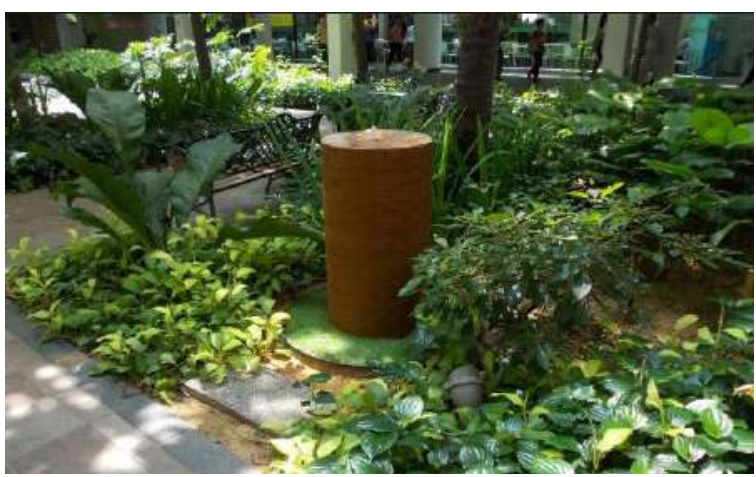

\section{- Jardines contemplativos}

Cuando el espacio y el presupuesto son limitados, la estructura puede optar por este tipo de jardín generalmente de pequeñas dimensiones, que no tiene un uso. Se los puede gozar solo a través de la vista estando sentados en una zona interna. Generalmente, son también de muy poco mantenimiento; pero el sentido del olfato y del oído no pueden apreciar lo expuesto, en este sentido, se vuelve frustrante ya que el mensaje que dan es "mirar y no tocar'.

\section{- Jardines contemplativos pero a uso limitado}

Son jardines que como en el caso anterior pueden encontrarse cerca de las entradas, en zonas de espera o corredores y, además de ser contemplativos, está permitido su uso para un número limitado de personas, generalmente presentan lugares donde poder sentarse. Indudablemente la parte negativa de esta tipología, y si es posible evitar, es crear el efecto pecera.

\subsection{Uso activo}

Simplemente caminar hacia una meta - Pasear

- Hacer ejercicios de rehabilitación elementales

- Para que los niños puedan jugar

- Hacer ejercicios de rehabilitación con la ayuda del personal y/o aparatos fijos

Poder realizar algún deporte

- Poder cultivar en huertas sobrelevadas

En este grupo podríamos incluir los llamados Jardines de rehabilitación y jardines 
terapéuticos. Son jardines donde el paciente puede experimentar una actividad ya sea física, mental, sensorial o de aprendizaje; tiene la oportunidad de compartir, aprender, enseñar y cuidar; superar desafíos físicos.

\section{Al servicio de una determinada patología médica}

Durante estos años se han aplicado las teorías de Ulrich y de Cooper Marcus especialmente en la creación de áreas verdes para determinados departamentos o estructuras médicas que se dedican al cuidado de patologías específicas, como por ejemplo:

\section{- Jardines para enfermos de Alzheimer $u$ otras enfermedades mentales}

En el caso de personas afectas de Alzheimer, podríamos mencionar el jardín del Centro Diurno de Chiavenna en donde teniendo en cuenta que el principal problema de esta patología es un compromiso de la percepción espacio-tiempo es muy importante realizar recorridos cerrados. Es decir, donde el ingreso y la salida sean una sola, sin caminos ciegos, crear un ambiente cerrado y seguro; pero sin que los pacientes lo perciban, con plantas que les hagan recordar su juventud, etiquetando plantas y herramientas de jardinería y colocado elementos que puedan ayudarles a recuperar la memoria.

\section{- Jardines para los centros de desintoxicación y rehabilitación de} drogas y alcohol

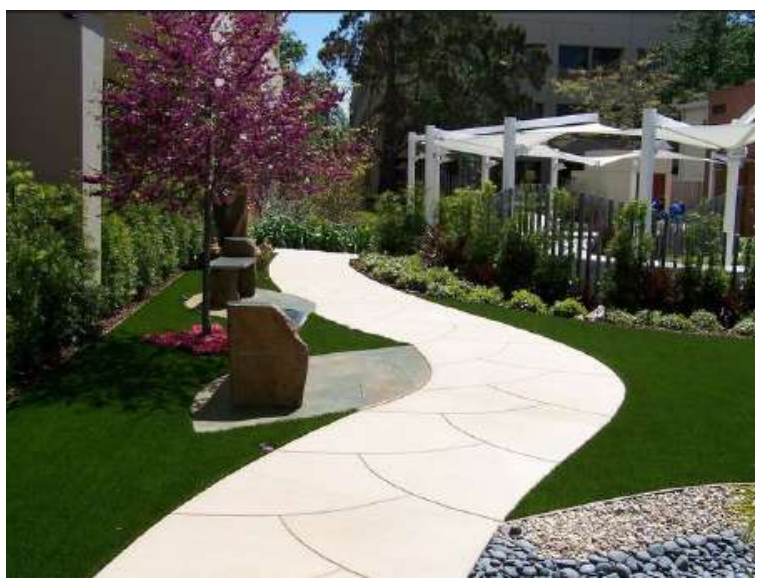

Serenity Garden, Scripps Memorial Hospital, San Diego, California USA

\section{- Jardines para enfermos de HIV/AIDS}

El Joel Schapner Memorial Graden al Cardinal Cook Hospital, (New York City), es un buen ejemplo de una terraza completamente inútil transformada en un oasis verde y colorada donde los pacientes pueden seguir un programa de horticultura y donde se ha puesto mucha atención en tener varias graduaciones de sombra ya que los medicamentos que toman muchos pacientes no les permite exponerse al sol. Esta terraza, que en el pasado estaba adecuada a la enfermedad, con los años se ha vuelto el espacio preferido por el personal hospitalario y es muy usada para eventos de recaudación de fondos. Con los años, la terraza fue reconstruida $y$, visto que la expectativa de vida de los enfermos se ha prolongado, el espacio ha sido equipado con un gimnasio al aire libre y un escenario para el karaoke.

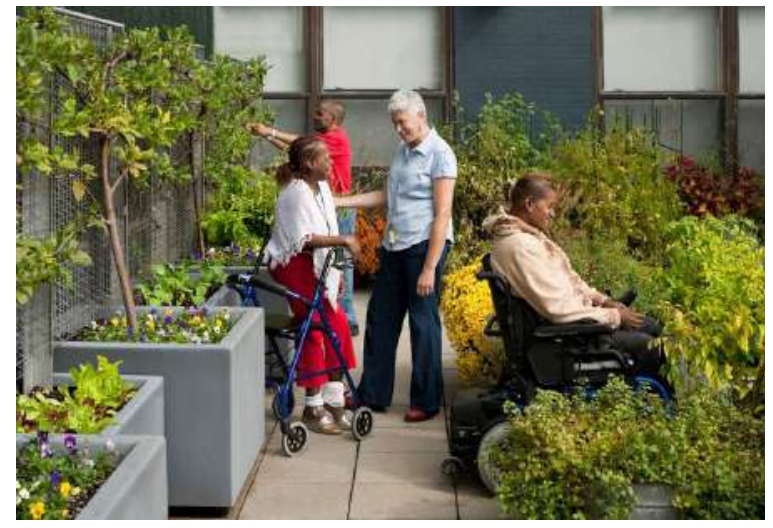

Joel Schapner Memorial Garden, Cardinal Cook Hospital, New York City

\section{- Jardines para enfermos de cáncer}

Aparte de las terrazas terapéuticas de Carrara mencionadas, otro ejemplo de éxito es el jardín del Mount Zion/ University of California San Francisco Medical Center, donde un triste patio de cemento fue transformado por un artistapaciente junto a un paisajista y otros enfermos. $\mathrm{El}$ jardín tiene un recorrido con bancos donde uno puede sentarse solo o acompañado, al sol o a la sombra, gozar de la vegetación y del sonido relajante del agua. Cuando fue terminado los pacientes junto a sus familiares participaron en un taller donde cada uno pudo contar su historia personal e imprimirla en una loceta que ahora decora el corredor que lleva hacia el jardín. 
- Jardines para diferentes tipos de descapacidad

- Jardines comunitarios para ancianos

- Jardines para departamentos pediátricos

En el Ospedale Pediatrico Meyer de Florencia, por ejemplo, es usada la hortoterapia. Esto ayuda mucho a los niños a mejorar su autoestima y son una oportunidad para satisfacer el instinto creativo reprimido durante la enfermedad. El pertenecer a un grupo, hace que adquieran seguridad en sí mismos, la labor de jardinería presupone control y autonomía que son elementos fundamentales para los enfermos crónicos. Además, cualquier medio que los distraiga de la evidente angustia que pasan los padres es una ayuda para poder sobrellevar la enfermedad.

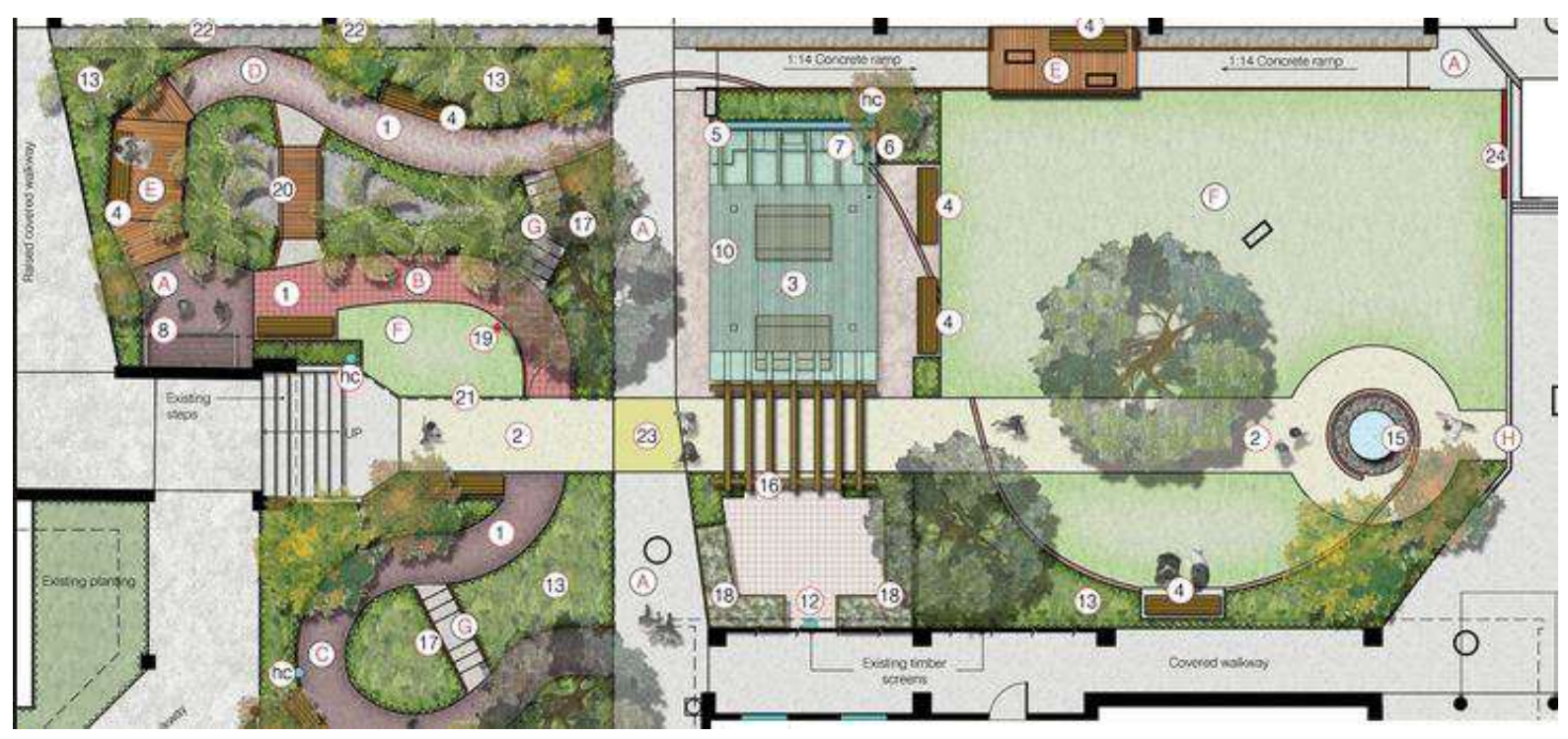

Maryborough Hospital Healing Garden

\section{Según el espacio a los que se tiene que adecuar}

Como bien nos podremos imaginar, aquí nos encontramos en el caso en el que la estructura sanitaria no ha sido planificada y diseñada desde un principio por un grupo de especialistas que incluya un paisajista $y$, por lo tanto, como hemos visto en ejemplos reportados anteriormente, se adapta una parte del edificio a un uso no previsto.

Los ocho tipos de espacios exteriores que menciono son los que Clare Cooper Marcus reporta en su estudio del 1994 de hospitales de la zona norte de California después de un atento estudio en cada uno de estos lugares.

\section{- Jardines entre edificios}

Es el caso más común de los viejos hospitales donde los jardines unen varios pabellones, comúnmente llamados parque o campus. Sirven como enlace entre los varios edificios y tienen lugares donde las personas se pueden sentar a esperar, conversar, comer. Es decir, tener una gran variedad de funciones $y$ englobar varias actividades. Generalmente los costos de mantenimiento son altos

\section{- Jardines alrededor del edificio}

Generalmente es el área que está delante o alrededor de un edificio y que lo separa de la circulación vial. El poder realizar un buen jardín en esta zona da al hospital una imagen confortable y familiar, como el jardín delantero de una casa, y hace que entre las habitaciones, oficinas y la calle se establezca una zona de privacidad. La desventaja más grande es que generalmente no cuenta con una acera o recorrido en donde poder colocar bancos y se vuelve limitado su uso activo, siendo prevalentemente pasivo $y$ de contemplación. 


\section{- Porche delantero}

Como en la mayor parte de tipología habitacional de los Estados Unidos, las casas como muchos hospitales cuenta con un porche delantero que facilitan la orientación de las personas que llegan al lugar, generalmente amoblada con bancos de manera que las personas que esperan puedan estar cómodas; además, brindan otros tipos de facilidades. Muchas veces, son el único lugar donde las personas pueden descansar o reunirse a conversar, así que se vuelve una zona muy congestionada de personas que salen, entran o permanecen allí. Se vuelve una zona conflictiva en el momento que, siendo exterior, se convierte en una zona de fumadores.

\section{- Jardines en el ingreso principal}

A diferencia de los dos anteriores, este ha sido diseñado y detallado para su uso. Es visible, accesible, da una imagen placentera del ambiente y ayuda a distraer a los pacientes ambulatorios.

\section{- Patios}

Crean un espacio semiprivado y seguro, a escala humana, que dependiendo donde esté ubicado puede ser de fácil visibilidad y accesibilidad; además, cuando en uno de los lados del edificio se ubica la cafetería, se puede volver en un agradable comedor abierto. El hecho que esté rodeado por edificios lo hace un espacio resguardado por el viento y en parte sombreado. La mayor desventaja está en la proporción, en cuanto a que si es de pequeña dimensión se tiene que resguardar la privacidad de los pacientes y, en muchos casos, se puede generar el llamado efecto 'pecera'.

\section{- Plaza}

Tener en un hospital como espacio exterior una plaza, es decir un espacio principal construido donde el verde se encuentra encajonado en maceteros, tendrá sus ventajas en cuanto a bajo costo de irrigación y manutención, facilidad de uso para las personas que usan sillas de ruedas o que tienen discapacidad motoras ya que pueden moverse sin mucho problema. Aunque, pierde el sentido de oasis que debería tener un jardín terapéutico y termina por parecerse más a un espacio englobado en un edificio de oficinas o un centro comercial.

\section{- Terrazas}

Áreas exteriores situadas indistintamente en los diferentes lugares del edificio y son de diferentes formas y dimensiones. Pueden ser usadas para alargar las visuales, con el inconveniente de que según donde estén ubicadas podrían resultar demasiado ventosas, calurosas, frías o sombreadas.

\section{- Techos jardín}

Estos espacios son un muy buen recurso para hospitales que se encuentran en centros urbanos muy densos y sin posibilidad de tener otras posibilidades de áreas exteriores. Generalmente ofrecen una visual amplia y una zona de uso privado, dependiendo de cuan altos y cercanos sean los edificios que los circundan.

\section{Según el tipo de diseño al que se inspira el paisajista}

Como todo proceso creativo, el diseño de un jardín, cualquiera sea el tipo, lugar o misión que tenga que cumplir, parte de un análisis del lugar, del medio ambiente, de las necesidades, del costo de realización, de manutención, etc. Sin embargo, en muchos casos, especialmente en Norte América, se han construido jardines escogiendo como fuentes de inspiración por ejemplo una metáfora: un jardín inspirado en antecedentes históricos, como por ejemplo el jardín a la italiana o el paisajístico inglés; el jardín doméstico, con porches y glorietas, muy adecuado para las personas ancianas que necesitan de un entorno que les resulte familiar; jardines inspirados a ciertas regiones geográficas, aunque puede ser muy limitante; jardines usados como museos de arte al aire libre donde exponer obras, siempre y cuando no sean impactantes para las personas o que provoquen reacciones negativas o de disgusto en el enfermo. 
Sin embargo es muy probable que este no sea enfoque justo, si es que no se considera al enfermo como punto focal del problema.

\section{ELEMENTOS PARA LA PROYECTACION}

A continuación, daré una serie de elementos que puedan ayudar al diseño de los espacios exteriores llamados "jardines terapéuticos":

1.- Ubicación y planificación

2.- Consideraciones generales

3.- Elementos naturales

4.- Elementos antrópicos

\section{Ubicación y planificación}

Como mencionaba anteriormente, sería apreciable y fundamental que el paisajista hiciese parte del grupo de proyección de la estructura sanitaria, pudiendo decidir la mejor ubicación, orientación, establecer la función, el acceso y la visibilidad del espacio verde de dicho complejo de manera que su realización y uso sean no solo un éxito sino que logre el objetivo para la cual ha sido creado.

En este sentido, es muy probable que si se trata de un complejo hospitalario de dimensiones considerables sea indispensable que cuente no solo con un espacio exterior que defina el ingreso, sino con uno adicional cerca de los ambulatorios de atención diurna, de uno cerca de la cafetería o comedor, de uno próximo al servicio del departamento de rehabilitación o pediatría, de un espacio exterior no solo contemplativo sino de uso general de todo el personal, pacientes incluidos y que tenga muy buenas vistas desde los dormitorios.

Si la estructura es de pequeñas dimensiones o especializada en el tratamiento de una determinada enfermedad o patología, el ambiente exterior será especifico para esto.

Dos son los elementos fundamentales que hay que tener en consideración y son: la visibilidad y la accesibilidad.

En cuanto a la primera, es fundamental que el espacio se vea, que se perciba que existe y que está allí. En el caso que eso no sea posible, es muy importante el uso de los carteles de orientación. Es importante también que el personal tenga visión de este y pueda de alguna manera ejercer un control del espacio y de las personas que lo usan. Por ejemplo, la estación de enfermeras podría estar ubicada cerca y/o tener una amplia visualización de la zona.

En cuanto a la accesibilidad, debe estar muy claro cómo poder acceder a dicho espacio. Las veredas, aceras, pasarelas o recorridos tienen que ser suficientemente anchos para permitir el tránsito de dos sillas de ruedas simultáneamente, la superficie debe ser lisa, no resbalosa, que permita la circulación de camillas y las ranuras entre materiales muy estrechas, de manera que no queden atrapadas las ruedas, los bastones o cualquier tipo de soporte que usen los pacientes.

\section{Consideraciones generales}

Partiendo de la premisa de que el éxito de un buen proyecto debe tener en cuenta que el espacio diseñado tiene que dar la oportunidad de acceso a todo tipo de personas, enferma o no, de hacer o no movimiento y ejercicio físico, tiene que dar la oportunidad de poder elegir entre tener privacidad o no y ayudar a experimentar un sentido de control; ya que desde el momento en que una persona enferma entra en un hospital pierde completamente el control de sus acciones (son otros los que deciden cuando tienes que comer, dormir, como vestirte $y$ cuando poder socializar $y$ ver parientes y amigos, generando de esta manera un alto grado de stress). El jardín les da la oportunidad de "escapar", de decidir cuánto y cuando poder estar en él, sea solo o en compañía de alguien, poder pasear o estar sentado al sol o a la sombra.

La familiaridad es un elemento que ayuda no solo al enfermo sino a todas las personas que pasan el día con ellos, tener un lugar acogedor con plantas y muebles a escala familiar ayuda a bajar el estrés.

El jardín tiene que ser un lugar donde reine la tranquilidad, escuchar moverse las hojas, correr el agua de un arroyo o una fuente, las 
campanillas eólicas o el cantar de las aves ayudan a recuperar un sentido de calma que no hay dentro de un hospital donde se escuchan los anuncios de los altoparlantes o el ruido de los carritos o los lamentos, sin contar los aparatos de aire acondicionado. Por esto, es importante que esté lejos del tráfico vial, estacionamientos, salidas de servicio y aterrizaje de helicópteros.

Tiene que ser un espacio en donde cualquier persona se sienta psicológicamente segura. Tan segura que le permita hasta echarse una siesta en el verde césped o en una banco. Para lograr un alto grado de seguridad física, será necesario equiparlo con corremanos, asientos cada determinados metros especialmente cerca de las puertas y asegurarse que el material usado en el piso no cree reflejos o deslumbre demasiado; que las bancos estén protegidos de las corrientes de aire, que unos estén en la sombra y otros en el sol, y sobre todo, que tengan respaldar y apoyabrazos.

Es necesario incentivar la socialización, ya que las personas más sociables tienen una mejor calidad de vida. Por esto, es aconsejable que las horas de visitas sean lo más prolongadas posibles. En este sentido, por ejemplo, el departamento de Oncologia del Ospedale di Carrara no tiene horarios para las visitas, las personas pueden ir y venir a cualquier hora del día. En esto, ayuda mucho que los muebles no solo sean fijos sino que se puedan mover según la necesidad de las personas.

Otro elemento importante es el poder gozar, cuanto posible, de obras de arte o poder escuchar buena música. Principios siempre aplicados en el Ospedale di Carrara, donde si bien sus dos terrazas terapéuticas eran pequeñas y los horarios de visitas ilimitados, todos los días se reúnen para cantar o tocar piezas musicales y cada mes con la participación de personajes importantes realizan conciertos; además, no faltan artistas que exponen sus obras en los corredores y salas de espera. En este sentido hay que tener mucha atención sobre el tipo de obra que se expone ya que el arte abstracto, si bien puede resultar interesante o indiferente a una persona sana o con bajo grado de estrés, en una persona enferma puede resultar amenazadora y causar mucha ansiedad. Por esta razón hay que considerar que la obra transmita siempre un mensaje positivo.

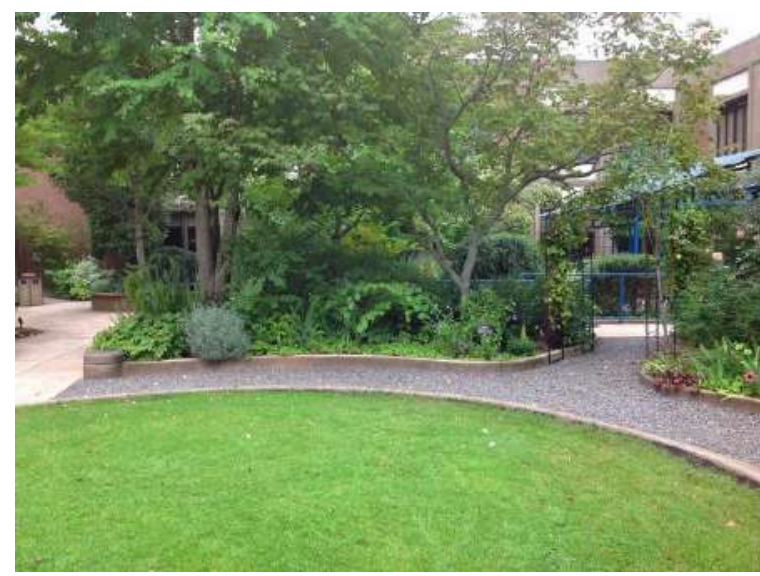

"BurnCenter Garden"Oregon

El diseño del jardín tiene que ser de fácil lectura, evitar que pueda crear confusión especialmente en estructuras sanitarias donde se tratan pacientes conimpedimentos psicológicos.

\section{Elementos naturales}

Siendo los elementos naturales lo fundamental, es recomendable que la proporción entre elementos vegetales y antrópicos sea de 7 a 3 para que la sensación sea efectivamente de un jardín y no de una plaza.

En cuanto a la elección de la vegetación es preferible que pertenezca al hábitat donde se construye la estructura sanitaria para que el enfermo mantenga una relación de familiaridad, como comentábamos antes, y facilitar el mantenimiento, evitando que permanezcan plantas con evidentes signos de mala ambientación, eliminando las partes muertas lo más pronto posible. Es aconsejable evitar las especies con alto potencial alergénico.

La vegetación tiene que ser exuberante y con gran variedad de colores, altamente llamativa. Árboles, arbustos y hierbas ornamentales con flores proporcionan un sentido de cambio estacional que refuerza la propia conciencia de los ritmos y los ciclos de la vida. Diferentes tipos de follaje, forma, color, que se muevan con la brisa, que den diferentes tipos de sombras 
creando una sensación de relajación. Escoger plantas que atraigan mariposas y aves que recuerden cuanto puede ser efímera la vida y la importancia de ésta.

De vital importancia asegurarse, si el jardín está al servicio de una tipología de enfermo en particular, como por ejemplo los que son tratados con quimioterapia, no soportan el perfume intenso de ciertas flores, por lo tanto, tener en cuenta la fragancia que éstas emiten es fundamental como que la ubicación del jardín no esté cerca de la cafetería o de la cocina del comedor/restaurante.

Asimismo, es importante crear una franja verde que separe el jardín de las ventanas que se asoman a él, ya sean de oficinas, consultorios o dormitorios de manera que se resguarde la privacidad que los usuarios en el interior.

En el caso de que se utilice como medio de terapia la horticultura, será importante que las huertas estén preferiblemente (o al menos buena parte de ellas) en maceteros altos de manera que, las personas no se tengan que agachar para poder trabajar y las que están en sillas de ruedas acceder fácilmente.

\section{.4. Elementos antrópicos}

Algunas indicaciones las he dado ya con anterioridad, como la más importante de todas es que estos espacios estén lejos de cualquier elemento antrópico que produzca ruidos, visuales y olores molestos.

Es muy importante, si es posible, introducir el elemento agua en cualquiera de sus formas, desde una pequeña fuente o tinaja para que se bañen las aves, hasta arroyos, cascadas, fuentes o pequeños lagos. El ruido del agua, la fauna y flora que se crean alrededor de ellas son, desde el punto de vista contemplativo, elementos desestresantes.

Si el espacio lo permite y la patología a la que está destinado el jardín, aparte de un recorrido definido y directo, crear un recorrido alternativo y serpenteado de manera que se puedan variar los puntos de vista y gozar de diferentes tipos de sombras.
Elegir un tipo de piso que sea liso pero no resbaladizo $y$, como mencionaba antes, sin obstáculos para las ruedas, bastones y camillas. Sobre todo, que no creen el efecto deslumbramiento; los fuertes reflejos del sol pueden crear problemas a personas que toman determinados medicamentos que sensibilizan el sentido de la vista.

En donde sea necesario, prever que el acceso al jardín sea lo suficientemente largo, como los mismos senderos, para que puedan acceder a ellos personas en sillas de ruedas o con las mismas camillas.

Las instalaciones eléctricas exteriores deben ser accesibles a los usuarios, para poder conectar computadoras, escuchar música o recargar celulares. Sería aconsejable que el uso del jardín perdure al oscurecer y para esto es necesario dotarlo de una adecuada iluminación artificial, especialmente si está lejos de los dormitorios.

En cuanto al mobiliario, se pueden prever elementos fijos para incentivar la socialización, como elementos movibles ya sean sillas, mesas y bancos como mínimo para dos personas. Además, elementos preferiblemente con respaldar y apoyabrazos. Se ha visto también que son de gran uso las grandes plataformas con asientos para que se puedan echar a tomar una siesta o simplemente poder apoyar los pies en alto. Asegurarse que la concentración visual esté dirigida hacia un punto focal apreciable o psicológicamente estimulante, evitando si es una terraza, por ejemplo que la balaustra o el parapeto la cubra.

Se puede prever el uso de sombrillas, pérgolas, estructuras que resguarden de las brisas u otro tipo de cobertura de fácil control y elementos que ayuden a controlar el clima para prolongar el uso del jardín el mayor tiempo posible durante todo el año. Debido a que muchos medicamentos impiden a las personas que se expongan al sol directo.

El mobiliario es preferible que sea de material cálido como la madera. Se ha visto que elementos en acero inoxidable, metal o plástico, muy en moda en los últimos años, 
no son muy bien aceptados ya que todo lo artificial dentro de la estructura sanitaria y es sinónimo de enfermedad.

\section{CONSIDERACIONES FINALES}

Los países donde se han realizado los estudios más avanzados son los del Norte de Europa, Inglaterra y USA. Hay que considerar que los jardines terapéuticos no son lugares estáticos, sino como las mismas plantas tienen un proceso dinámico, se transforma y es por esta razón importante que se aplique después de su construcción el POE (post ocupancy evaluation) que ayuda a medir las reacciones que el espacio ha producido en las personas, si ha satisfecho a sus necesidades y en qué medida se puede mejorar.

El primer POE se realizó en el 1994 y lo hicieron Cooper Marcus y Barnes estudiando 4 jardines de la Bahía de San Francisco, como mencioné anteriormente. Los elementos, que nacen de este $\mathrm{POE}, \mathrm{y}$ que determinan cuán importante y fundamental sea considerarlos para un buen diseño son: el movimiento, la privacidad, tranquilidad, socialización, control de la visibilidad, familiaridad y la distracción que da la naturaleza. Es muy probable que si sigue la investigación y con los tiempos que cambian se lleguen a implementar estos elementos con los nuevos POE.

Gracias a estos estudios es que se ha llegado a la conclusión de que el proceso de diseño de los mismos es participativo, debe ser realizado por un equipo multidisciplinar, en donde colaboren también médicos y es fundamental tener la opinión de los pacientes, y si posible de los ex-pacientes para que puedan explicar sus necesidades y exigencias, así como del personal sanitario que trabaja en él. Este es el concepto del IHS (Integrated Healthcare Strategies)

Hoy existe el EBD (Evidence-based design) metodología que ayuda en el proceso de diseño, apoyándolo en la racionalización de los costes de mantenimiento del sistema sanitario, basado entre otras cosas por los resultados obtenidos de otros POE y el IHS.
En USA, el Center for Health Design ayuda no solo en la proyección de clínicas, hospitales y sus servicios como los jardines terapéuticos, sino que certifica con el EDAC (Evidencebased Design Accreditation and Certification) el proyecto en el caso se haya usado el EBD como metodología de diseño.

Calidad ambiental y buen mantenimiento contribuyen a una buena salud no solo de las plantas sino de toda la comunidad. Un buen mantenimiento de las plantas es muy importante también desde el punto de vista de la seguridad, aparte del potencial terapéutico, es indudable que árboles, arbustos y hierbas necesitan de un mayor mantenimiento que un simple patio o plaza, sin embargo, es el elemento verde el que tiene importancia terapéutica.

Dentro del mantenimiento, sería aconsejable que los elementos vegetales fuesen tratados con productos naturales, desde la fertilización hasta la lucha contra las enfermedades evitando el uso de productos químicos.

En el momento en que el presupuesto no lo permita o sea muy restringido, optar, con la colaboración de voluntarios, ya sea para el mantenimiento como para que los pacientes puedan acceder a él de manera controlada si fuese necesario

Educar al personal sanitario sobre la importancia del uso, ubicación y control del jardín y para que a su vez ellos divulguen $e$ incentiven su uso a las personas que llegan a la estructura sanitaria, son de gran ayuda para lograr el éxito del proyecto. Así como, dejar que dichos espacios sean siempre accesibles a las personas a cualquier hora y en cualquier momento.

Los jardines terapéuticos son un recurso que debe ser usado con el máximo beneficio, promoviendo su conocimiento y el alto grado de influencia positiva que aportan a toda la comunidad hospitalaria. Estos podrían considerarse un componente importante de las mismas, siempre y cuando se les reconozca su validez terapéutica como hemos demostrado. 
Se le podría considerar dentro de una buena estrategia de marketing empresarial. $\mathrm{Si}$ el concepto es bien asimilado por la administración, el problema económico y el recaudar fondos para la construcción y mantenimiento no representarían un problema, como lo ha sido en muchos casos.

Cada país tiene sus leyes. En Italia, por ejemplo, ya existe una ley de 1939 que prevé dejar $15-20 \mathrm{~m}^{2}$ de área libre por cada cama que tiene para ser asignada a jardín. Lógicamente no ha sido aplicada por mucho tiempo o en todo caso, esa área nunca ha sido diseñada específicamente para cumplir la función de jardín terapéutico.

La ley peruana en sus Normas Técnicas para Proyectos de Arquitectura Hospitalaria (marzo 1996) prevé que se debe dejar como área libre destinada a verde el $50 \%$ del terreno, para que uso especifico? Generalmente estas áreas son mal relacionadas con el edificio y son áreas ocasionales que han sobrado. En realidad, de todos modos, son áreas verdes que ya existen y que de alguna manera se podrían reproyectar y darles un uso convirtiéndolas en jardines terapéuticos. 


\section{REFERENCIAS}

Bonati, P. and Valla, P. (2000) "Quali strumenti per la prevenzione della contenzione? Lipotesi ambientale". In: Belloi, L., Valgimigli, C., La notte dell'assistenza - 'I vecchi legati': quali alternative?, Franco Angeli Editore, Milano.

Burton, A. (2014) "Gardens that take care of us", in www.thelancet.com/neurology, Vol. 13, May 2014. 447-448.

Cooper Marcus, C. (2007) "Healing Gardens in Hospitals", IDRP Interdisciplinary Design and Research, available at http://www.idrp.wsu.edu/, Vol. 1, Issue 1: Design and Health.

Cooper Marcus, C. "Gardens and health", WCDH 2000, International Academy for Design \& Health (IADH), available at http://www.designandhealth.com/uploaded/documents/Publications/ Papers/Clare-Cooper-Marcus-WCDH2000.pdf, (accessed on January 2015).

Cooper Marcus, C. and Barnes, M. (1995) Gardens in Health Care Facilities: Uses, Therapeutic Benefits, and Design Considerations, Martinez, CA: The Center of Health Design.

Cooper Marcus, C. and Barnes, M. Eds. (1999) Healing Gardens: Therapeutic Benefits and Design Recommendations, New York: John Wiley \& Sons.

Cooper Marcus, C. and Sachs, N.A. (2013) Therapeutic Landscapes: An Evidence-Based Approach to Designing Healing Gardens and Restorative Outdoor Spaces, New York: John Wiley and Sons.

Del Nord, R. (2002) Architettura per l'Alzheimer: il malato di Alzheimer e l'ambiente, Voll.1/2, Centro Stampa Regione Toscana, Firenze.

Del Nord, R. and Peretti, G. (2012) Lumanizzazione degli spazi di cura. Linee guida, Ministero della Salute - TESIS, Firenze.

Erickson, M. S. (2012) "Restorative Garden Design: Enhancing wellness through healing spaces", JAD Art and Design Discourse Journal, No. 2. 89-102.

Gerlach-Spriggs, N., Kaufman, R. E. and Warner, S. B. Jr. (1998) Restorative Gardens: The Healing Landscape. New Haven, CT and London: Yale University Press.

Nedučin, D., Krklješ, M. and Kurtovic-Folic, N. (2010) "Hospital Outdoor Spaces - Therapeutic Benefits And Design Considerations", Facta Universitatis Series: Architecture and Civil Engineering, Vol. 8, No 3. 293-305.

Pedretti Burls, A. (2008) "Seeking Nature: A Contemporary Therapeutic Environment", Therapeutic Communities, 29, 3, autumn. 228-244.

Sherman, S.A., Varni, J. W., Ulrich Roger, S. and Malcarne, V. L. (2005), "Postoccupancy evaluation of healing gardens in a pediatric cancer center", Landscape and Urban Planning, Vol. 73, pp. 167-183.

Stigsdotter, U. A. and Grahn, P. (2002), "What makes a garden a healing garden". Journal of Therapeutic Horticulture. 60-68. 
Tartarini, R. et alii (2011) "Uso del Verde per scopi terapeutici Terrazze terapeutiche in Oncologia a Carrara", in Galileo 200, Mar-Apr. 10-12.

Ulrich, R. (1984) "View Through a Window May Influence Recovery from Surgery", Science, vol. 224. $420-421$.

Valente, R. (2014) "Sustainable Sites Initiatives: US updated rating criteria for open spaces design", in Techne, n'8. 70-80.

Valla, P. (2005) "Architetture e giardini come strumento terapeutico", Milano, Guerrini Editore.

Fecha de recepción: 24 de julio 2015

Fecha de aceptación: 20 de agosto 2015 